\title{
A Comparative Study on Various Edge Detection Techniques used for the Identification of Penaeid Prawn Species
}

\author{
V. Sucharita \\ Dept. of Computer Science \& Engg. \\ Audisankara College of Engg. \& Tech. \\ Gudur, INDIA
}

\author{
S. Jyothi \\ Dept. of Computer Science \\ SPMVV \\ Tirupati, INDIA
}

\author{
D.M. Mamatha \\ Dept. of Sericulture \\ SPMVV \\ Tirupati, INDIA
}

\begin{abstract}
Image recognition is a challenging problem. The identification of prawn species can be done using Image Processing. Each species of the prawn has got its own distinctive patterns which enable the various researchers to perform some research on it and to accurately classify the prawns. In this paper the fundamental concepts of different edge detection techniques are to be studied and apply the best techniques in identifying the Penaeid Prawn species type. This paper presents a comparative study of various edge detection techniques such as Sobel, Robert's operator, Prewitt's cross operator and Canny's edge detector. For the Prawn species identification image processing is used in which edge detection is the primary function. Particular edge detection method can be employed to characterize edges to represent the image for further implementation.
\end{abstract}

\section{General Terms}

Feature extraction, Penaeid prawn, classification.

\section{Keywords}

Edge detection, prawn, image processing, species

\section{INTRODUCTION}

The prawns of India belong to three major families, namely Pedaeidae, Sergestidae and Palaemonidae of the decapod groups. Prawns are among the most popular types of seafood. With the increase in demand for seafood, the marine aquaculture has become one of the growing industries in the world wide. Various types of prawns are found in both brakish water and fresh water. Freshwater prawn farming is an aquaculture business was mainly designed to raise and produce freshwater prawns for human consumption. The penaeid prawns include here are three categories namely Penaeus Monodon, Penaeus Indicus and Vannamei.

The images of different prawns are isolated from one another and from the background. The physical differences between the types of prawns are length, body mass, width, pleopod, pereiopod and tail. The automatic recognition of prawn species by their patterns is a very interesting field. Some areas of the aquaculture particularly fish family have been benefited by the automation and monitoring equipment. But these advances are not implemented in the prawn aquaculture. The need for computer based prawn species recognition systems that can automatically recognize species of prawns from digital images is expected to increase in the near future. In general it is not easy for a human being to inspect and recognize the bulky amount of prawn species and more over it is extremely cost effective. So automatic classification of the prawn species is necessary to overcome the errors caused by manual sorting of prawn species which is completely based on the human expertise. The goal is to develop commercially possible system that allows the farmers of the prawn to reduce the labour costs. For this, the usage of image analysis is proposed for automation. The main idea of this paper is to make a comparative study on various edge detection techniques which gives a way for selecting one suitable technique for identifying the species of Prawn. Edge detection is one of the mostly used operations in image analysis. The reason for this is that edges form the outline of an object[2]. An edge is defined as the boundary between an object and background, and also indicates the boundary between overlapping objects. Since computer vision involves the recognition and classification of objects in an image, the edge detections is very much essential tool. For an Image, Three basic steps involved in the selection of the edge detection [4] are:

1. Noisy Environment: It is very difficult to detect edges in the noisy environment because the edges and noise have high-frequency count.

2. The orientation of the edges

3. Extracting of all the edge points that are potential candidates to become edge point.

To perform edge detection there are many techniques. All the techniques are grouped into two categories: Gradient and Laplacian. Here the various edge detection techniques are studied and analyzed and also visual comparisons have been done.

\section{PROBLEM DOMAIN}

Prawn species recognition and classification is an active area in aquaculture. Some features may vary among different prawn species. Image acquisition process can be affected by noise and considered as a potential research in utilizing the existing technology for encouraging and pushing the aquaculture researches ahead. Although advancements have been made in the areas of developing real time data collection and on improving range solutions, existing systems are still limited in their ability to detect or classify prawns. There is a difficulty in identifying the different species of prawns. The classification is made by analyzing prawn with the various features as shown in Figure1. The various species of prawns with different morphological features look identical[12]. It has to be processed using the image processing. The image processing concept mainly deals with three aspects. The first is edge detection of the prawn, followed by feature extraction and then training. To do image processing with computers, the more suitable images are to be created for the people to identify and examine. Then the computer can identify the image. Edge is the basic feature of an image. It contains all the internal information of the image. There are so many edge detectors, 
The main problems is that edge detectors work differently. Some of them may take more time when compared to other. Some edge detectors finds more edges when compared to other. So the edge detection for image mainly depends on the noise, intensity, brightness, and blur. By working with different edge detectors for the same image the actual difference can be found. This paper pertains with study and comparison of various edge detection techniques for a single image and applying the best technique for identifying the species of prawn. The system has to recognize the isolated pattern of prawn which is consisting of its morphological features by which it is identified. As the system acquire an image consisting pattern of prawns then the image will be processed into several phases such as edge detection, feature extraction and then training for identifying the prawn with morphological feature extraction[1] before recognizing the pattern of the Prawn.

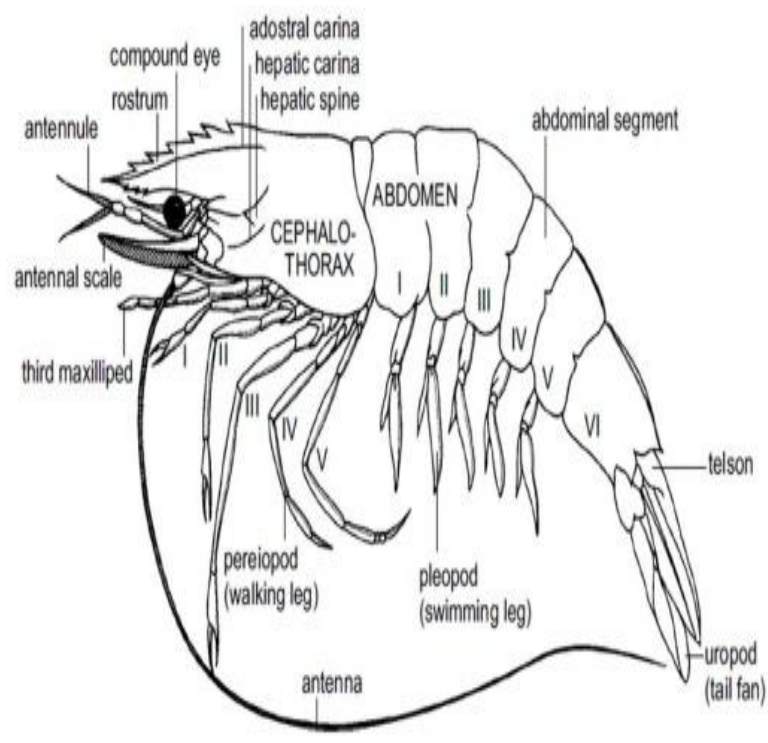

Figure 1: Morphological Features

\section{EDGE DETECTION TECHNIQUES}

Edge detection is the problem of fundamental importance in image analysis. Edges characterize the object boundaries and are useful for segmentation, and identification of objects. Edges in an image are the locations of the pixel with the rapid changes in the gray levels. If there is a continuous image then the derivative of the image $f(x, y)$ assumes a local maximum in the direction of the edge[8]. Therefore, one of the edge detection techniques is to measure the gradient of $\mathrm{f}$ in particular location. This is performed by using a gradient operator. Such operators are also called masks. It provides finite-difference approximations of the orthogonal gradient vector fx and fy Gradient Operator [11].

The gradient of an image $f(x, y)$ at location $(x, y)$ is defined as the vector.

$$
\Delta f=\operatorname{grad}(f)=\left[\frac{G x}{G y}\right]=\left[\begin{array}{l}
\frac{\partial f}{\partial x} \\
\frac{\partial f}{\partial y}
\end{array}\right]
$$

This vector always points in the direction of greatest rate of change of $\mathrm{f}$ at location $(x, y)$. The magnitude of vector can be given as

$$
M(x, y)=\sqrt{\left(G_{x}^{2}+G_{y}^{2}\right)}
$$

There are different ways to perform edge detection. They are Sobel, Prewitt, Robert and Canny. All these four edge detection techniques are implemented in MATLAB7.3 on prawn images [6].

\subsection{Sobel Edge Detector}

The operator consists of the pair of $3 \times 3$ convolution kernels in Sobel Edge detector as shown in Table1. One kernel is simply the other rotated by $90^{\circ}$. The two convolution masks $G_{x}$ and $G_{y}$ is used by this sobel operator which as shown in Table1. This can be joined together for finding the absolute magnitude and the orientation of the gradient. Gradient magnitude given by

$$
|G|=\sqrt{\left(G_{x}^{2}+G_{y}^{2}\right)}
$$

Table 1. Masks used by Sobel

\begin{tabular}{|c|c|c|}
\hline-1 & 0 & 1 \\
\hline 2 & 0 & 2 \\
\hline-1 & 0 & 1 \\
\hline
\end{tabular}

\begin{tabular}{|c|c|c|}
\hline-1 & -2 & -1 \\
\hline 0 & 0 & 0 \\
\hline 1 & 2 & 1 \\
\hline
\end{tabular}

$$
\begin{aligned}
& G_{x}=-1 z_{1}+1 z_{3}-2 z_{4}+2 z_{6}-1 z_{7}+1 z_{9} \\
& G_{y}=1 z_{1}+2 z_{2}+1 z_{3}-1 z_{7}-2 z_{8}-1 z_{9}
\end{aligned}
$$

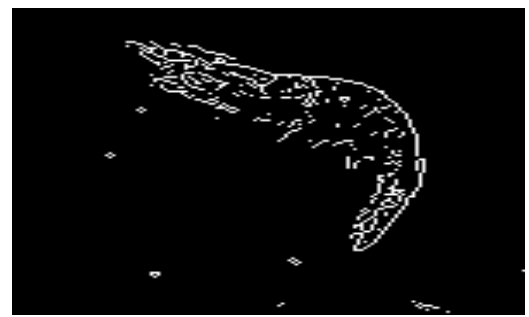

a) Vannamei

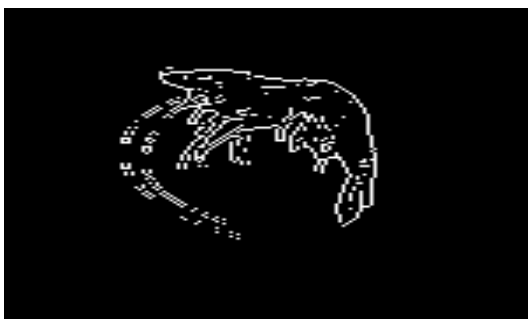

b) Monodon

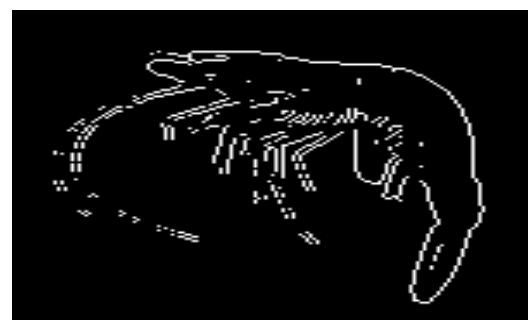

c) Indicus

Figure2: Sobel Filter 
Where $\mathrm{Zi}, \mathrm{i}=1,2, ., 9$ are intensity levels of each pixel in the convolution window, the magnitude of the gradient is threshold finally. Sobel operator is effective method to find the edges in image. The sobel operator is used mostly for detecting vertical and horizontal edges[3][5]. The edges detected by this sobel operator may not be suitable for all the applications[7]. As per the Sobel Edge Detection the images of the three species of Prawn are shown in Figure2.

\subsection{Prewitt's edge detector}

The Prewitt's edge detector uses the 3 X 3 convolution mask, which is slightly different from the Sobel edge detector. The convolution mask is used for smoothing the image.

Table 2. Masks used by Prewitt

\begin{tabular}{|c|c|c|}
\hline-1 & 0 & +1 \\
\hline-1 & 0 & +1 \\
\hline-1 & 0 & +1 \\
\hline
\end{tabular}

\begin{tabular}{|c|c|c|}
\hline+1 & +1 & +1 \\
\hline 0 & 0 & 0 \\
\hline-1 & -1 & -1 \\
\hline
\end{tabular}

The prewitt's edge detector is same as that of sobel operator and is also used for detecting horizontal and vertical edges in the image [3]. The kernel used in the prewitt's operator is shown in Table2.

To produce measurements of gradient component in each orientation $\left(G_{x}\right.$ and $\left.G_{y}\right)$. To find absolute magnitude and orientation of the gradient they can be combined together. The kernels can be applied to the input image. Prewitt Edge Detection of the three species of Prawn is shown in Figure3.

\subsection{Robert's cross operator}

The Roberts cross operator has $2 \times 2$ convolution kernels. It does fast computing. One kernel is simply the other rotated by $90^{\circ}$. These kernels are designed to respond maximally to edges running at $45^{\circ}$ to the pixel grid. One kernel for each of the two perpendicular orientations. The Roberts Cross operator performs a simple and quick to compute, 2D spatial gradient measurement on an image. Pixels at each point in the output represent the expected absolute magnitude of the spatial gradient of the input image at that point. The convolution masks for Roberts operator is shown in Table 3.
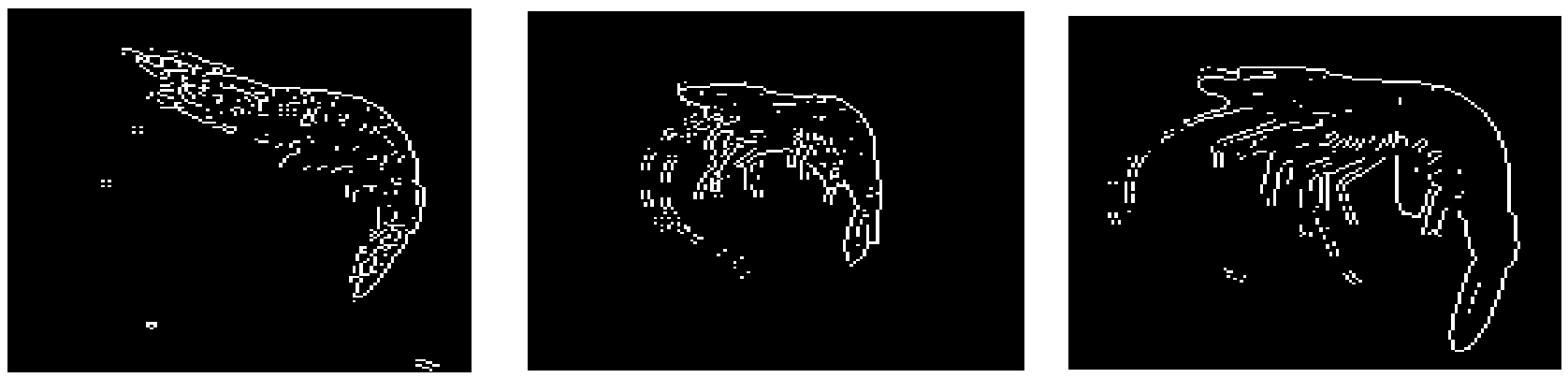

a) Vannamei

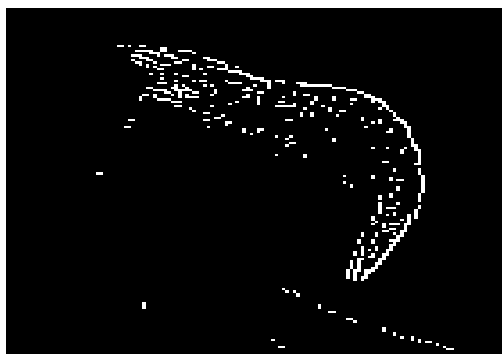

a) Vannamei

Table 3. Masks used by Roberts

\begin{tabular}{|c|c|}
\hline+1 & 0 \\
\hline 0 & -1 \\
\hline
\end{tabular}

\begin{tabular}{|c|c|}
\hline 0 & +1 \\
\hline-1 & 0 \\
\hline
\end{tabular}

b) Monodon

Figure3: Prewitt Filter

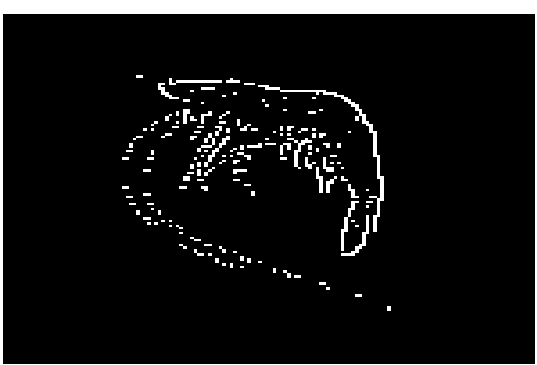

b) Monodon

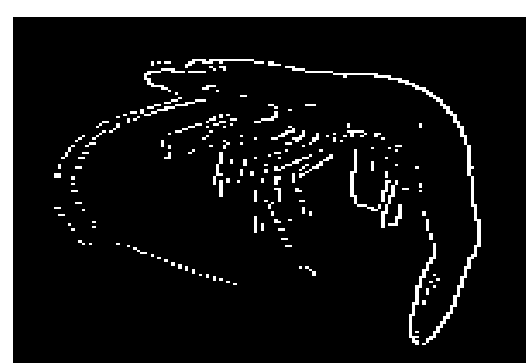

c) Indicus
Figure4: Roberts Filter c) Indicus
The Roberts cross detector has only one disadvantage that it fails to detect few of the edges. These masks are mainly designed to respond maximally to edges running at $45^{\circ}$ to the pixel grid, one kernel for each of the two perpendicular orientations. The angle of orientation which gives spatial gradient for an edge is $\theta=\arctan (\mathrm{Gy} / \mathrm{Gx})-3 \pi / 4$. As per the 
Robert Edge Detection the images of various species of the Prawn is shown as in Figure4.

\subsection{Canny's Edge Detector}

The Canny edge detector is also known to be an optimal edge detector [9], which satisfies all of the performance criteria. The steps for algorithm are as follows:

1. The first step in this detector is to filter out noise in the image by using a Gaussian smoothing filter[10].

$\mathrm{g}(\mathrm{m}, \mathrm{n})=\mathrm{G} \sigma(\mathrm{m}, \mathrm{n}) * \mathrm{f}(\mathrm{m}, \mathrm{n})$

Where $G \sigma=\frac{1}{\sqrt{2 \pi \sigma^{2}}} \exp \left[-\frac{m^{2}+n^{2}}{2 \sigma^{2}}\right]$

2. The second step in this edge detector is to find the edge strength in the smoothed image by computing the gradient of an image, which helps to point out where the actual edge is located. Gradient $\mathrm{g}(\mathrm{m}, \mathrm{n})$ is computed using

$$
M(m, n)=\sqrt{g^{2} m(m, n)+g^{2} n(m, n)}
$$

and

$$
\theta=\tan ^{-1}\left[\frac{g n(m, n)}{g m(m, n)}\right]
$$

3.The Threshhold M

$$
M t(m, n)=\left\{\begin{array}{c}
M(m, n) \\
0
\end{array} \text { if } M(m, n) T \text { otherwise } 0\right.
$$

4. The final step is to thin down the edges by tracking along the edge in the edge direction and set the pixel that is not at the maximum to be 0 , which is called non-maximum suppression[7]. Finally the edges are detected and linked using pixel connectivity and double thresholding that is, if the magnitude is above the high threshold, then it is considered as an edge. If the magnitude is below the low threshold, then it is considered to be a non-edge.[7].

This edge detector has the advantage that maximum edges get detected by using this edge detector. As per the Canny Edge Detection the images of the Prawns is shown as in Figure5.

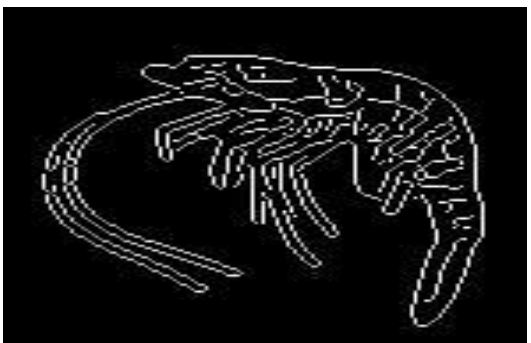

Vannamei

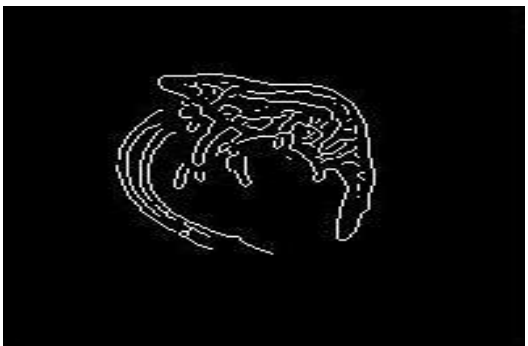

Monodon

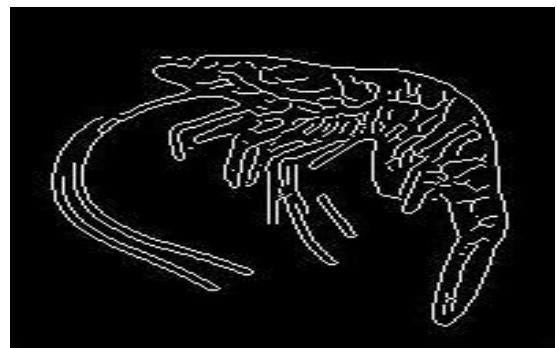

Indicus

Figure5: Canny Filter

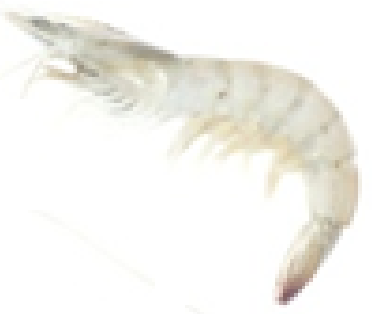

a) Vannamei

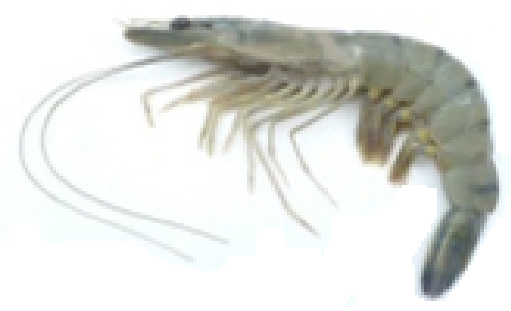

b) Monodon

Figure6: Prawn images used for Edge Detection

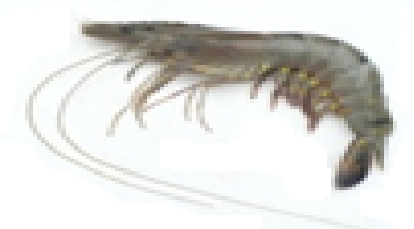

c) Indicus

\section{RESULTS AND DISCUSSION}

For visual comparison of edge detectors the images of Monodon, Indicus and vannamei are chosen which are the species of Paenaid prawns with noise are applied to the different edge detectors such as Sobel, Prewitt, Robert and Canny. The original images used for the analysis of edge detection are shown in Figure6. In the image recognition edge detection is the basic point, so the differences between various edge detection techniques are to be known. In this paper various edge detection techniques are studied and analyzed. The Sobel method finds the edges using the Sobel approximation to the derivative. Sobel method is good for detecting vertical and horizontal edges. The difficulty it had with certain prawn features such as rostrum, pereiopod and 
pleopod, mouth, antennules and tails are noticed. The Prewitt edge detector finds edges using Prewitt approximation to the derivative. It returns the edges at those points where the gradient of I is maximum. The Prewitt edge detector has the drawback of being very sensitive to the noise. The size of the filter for an image cannot be adopted. Roberts cross operator is unable to detect all the edges of the prawn, it suffers from miss mapping some of the lines. The Canny method finds all the edges by seeing for local maxima of the gradient of I. By using the derivative of a Gaussian filter the gradient is calculated. The performance of the canny is very good and depends on the adjustable parameters, $\sigma$ which is standard deviation and the method also uses thresholds for detecting strong and weak edges, and considers the weak edges in the output only if they are connected to strong edges. $\sigma$ also controls the Gaussian filter size. Canny is also able to detect the maximum number of edges, circular edges and edges at the corner. Edge detection of all four types were compared as shown in the Figure 7. Canny yielded the best results.

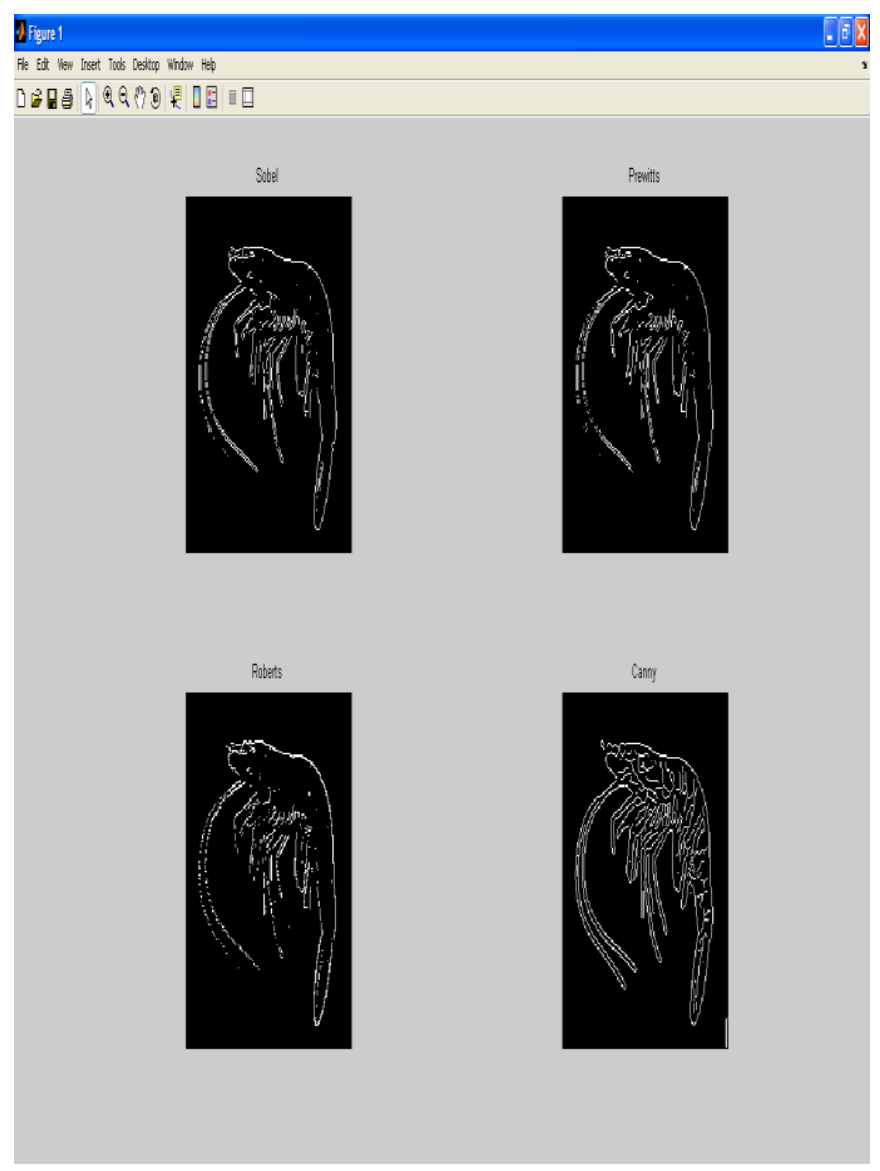

Figure 7: Various Edge Detection Filters

\section{CONCLUSION}

After performing a comparative study on different edge detection techniques we can say that Each approach has got its own Pros and Cons in different areas. But the experimental and visual comparisons on different approaches show which approach is best suitable for Prawn image type. The software was implemented using the MATLAB 7.3 although, all the techniques are nearly same for the noiseless image, but for practical applications. Canny edge detector is able to identify maximum number of the edges along with circular edges and also edges at the corner. Using Canny the we can make modifications to the existing algorithm by adjusting the parameters which can be able to adapt different environments.

\section{ACKNOWLEDGEMENTS}

Authors would like to thank DBT, New Delhi for sanctioning the Project. Currently this work is carried out under DBT Project.

\section{REFERENCES}

[1] Dr.T.Kannupandi and ,Dr.P.Soundarapandian and Dr.N.Rajendran "Prawns and Shrimps",Centre of Advanced Study in Mariane Biology,Annalamai University.

[2] M.B. Ahmad and T.S. Choi , Local Threshold and Boolean Function Based Edge Detection, IEEE Transactions on Consumer Electronics, Vol. 45, No 3.August 1999

[3] Raman Maini \& Dr. Himanshu Aggarwal, International Journal of Image Processing (IJIP), Volume (3) : Issue (1).

[4] Malay K. Pakira, "digital image processing and pattern recognition".

[5] R. C. Gonzalzez and woods "digital image processing" third edition.

[6] MATLAB Neural Network toolbox.

[7] Yuan-Hui $\mathrm{Yu}$,Chin-Chen Chang, "A new edge detection approach based on image context analysis" Image and Vision Computing 24 (2006) 10901102(www.sciencedirect.com)

[8] Canny,J.," A computational Approach to Edge Detection", IEEE Trans. Pattern Analysis and Machine Intelligence, Vol. 8:679-714, November 1986.

[9] S. Price, "Edges: The Canny Edge Detector", July 4,1996.http://homepages.inf.ed.ac.uk/rbf/CVonline/LOC AL_COPIES/MARBLE/low/edges/canny.html

[10] Narendra.VG and Hareesh.KS,"study and comparison of various image edge detection techniques used in quality inspection and evaluation of agricultural and food products by compter vision., Vol 4,No 2.June 2011.

[11] Meghana D.More and G.K.Andurkar, Edge Detection techniques:a comparative approach" proceedings of NCACC'12' April21,2012

[12] C.B.T Van de Braak "Haemocytic defence in black tiger shrimp(Penaeus monodon)" Ph.D thesis Wageningen university. 